\title{
A COHESION BASED FRIEND RECOMMENDATION SYSTEM
}

\author{
Shamsu Shehu \\ Al-qalam University, Katsina, Nigeria
}

\begin{abstract}
Social network sites have attracted millions of users with the social revolution in Web2.0. A social network is composed by communities of individuals or organizations that are connected by a common interest. Online social networking sites like Twitter, Facebook and Orkut are among the most visited sites in the Internet chew, (2008). In the social network sites, a user can register other users as friends and enjoy communication. However, the large amount of online users and their diverse and dynamic interests possess great challenges to support such a novel feature in online social networks kwon, (2010). In this work, we design a general friend recommendation framework based on cohesion after analyzing the current method of friend recommendation. The main idea of the proposed method is consisted of the following stagesmeasuring the link strength in a network and find out possible link on this network that is yet to be established; detecting communities among the network using modularity and recommending friends. Considering the noticeable attraction of users to social networking sites, lots of research has been carried out to take advantage of the users information available in these sites. Knowledge mining techniques have been developed in order to extract valuable pieces of information from the users' activities. This paper deals with a methodology to generate a social graph of users' actions and predict the future social activities of the users based upon the existing relationships. This graph is updated dynamically based on the changes in the selected social network. The forecasting performed is based upon some predefined rules applied on the graph.
\end{abstract}

\section{INTRODUCTION}

Social Networking Sites (SNS) are the dominating entities in the modern web Hassan, (2006) Online friendship is now similarly appealing to people as offline friendship if not more. People tend to enjoy the fellowship of their real life friends in a virtual world. At the same time they are interested in making online friends. But With the rapid growth of SNS's resulting in information overload people are in dilemmas to choose the right friend and rightly so. Potential choice of friends is influenced by many intrinsic as well as exogenous factors with respect to SNS 's. And with the invent of all types of virtual communication tools it is becoming more unpredictable who will like whom. This is of more importance to search engine companies and SNS 's because the increase of people of similar minds expedites the probability of buying similar products. Hence Product advertisement which is the driving force of modern e-commerce gets easier Hashima, (2006). The recent surge of research in recommendation algorithms is therefore not surprising. Popular movie recommendation site Netflix (www.netflix.com) had even conducted competition with $\$ 1 \mathrm{M}$ grand prize for a $10 \%$ improvement on their movie recommendation algorithm Silva, (2010).However, recommendation of products and recommendation of human beings areas

Dhinaharan Nagamalai et al. (Eds) : AIS, CSIT, IPPR, IPDCA - 2017

pp. 109-124, 2017. (C) CS \& IT-CSCP 2017

DOI : $10.5121 /$ csit.2017.71010 
disparate as can be. Historically there has been two main recommendation algorithms content based and collaborative algorithm. Content based algorithm requires textual information as its name suggests and recommends websites newspaper articles and other contents. Collaborative based algorithm recommends products to a user which it believe shave been light by similar users. Both of these algorithms have yielded unsatisfactory results in friend recommendation because of their lack of intuition in judging rational beings.

In the last few years, social networks have been increasing in both size and services. Social networking services (SNSs) such as Facebook, MySpace, Twitter, Flickr, YouTube and Orkut are growing in popularity and importance and to some extent they are also contributing to a change in human social behavior Salim, (2003). Online social friends are more influencing and people are now eager to make friendship with their same mentality. As the size is growing, it is very difficult to find out good friends and sometimes the user gets negative result. So the user of this social networking site expects that the system administrator will help them by friend recommendation system. Many researches are going on this point to find out recommendation system on various ways. We also try to improve the recommendation system on the basis of cohesiveness of friendship.

\subsection{Social Networking}

With the advent of Web 2.0, social computing has emerged as one of the hot research topics recently. It involves the collecting, extracting, accessing, processing, computing and visualizing of social signals and information. SNSs are an online phenomenon which provides social network based services to support easy message posting, information sharing and inter-friend communication Guy, (2009).SNA has its origins in both social science and in the broader fields of network Analysis and graph theory. Network analysis concerns itself with the formulation and solution of problems that have a network structure; such structure is usually captured in a graph. Graph theory provides a set of abstract concepts and methods for the analysis of graphs.

A social network is a set of people or groups of people with some pattern of contacts or interactions between them Dominic, (2010). The patterns of friendships between individuals, business relationships between companies, and intermarriages between families are all examples of networks that have been studied in the past. Social Network Sites are defined as web-based services that allow individuals to(1) construct a public or semi-public profile within a bounded system, (2) articulate a list of other users with whom they share a connection, and (3) view and traverse their list of connections and those made by others within the system Dumas, (2003). Social Networking sites(SNS's) provide users with opportunity to connect with their offline friends as well as making new friends with latent ties who otherwise would never have met them. They also supplement their relationships with close relations and help to maintain the social capital Kim, (2010). People tend to trust the opinions of friends they know rather than the opinions of strangers.

\subsection{Key Terminologies of Social Network}

As social networking sites like facebook, twitter, google plus etc are getting popular day by day, social networking analysis are becoming an important subject for the researcher. There are some basic terminologies in social networking analysis. Some of them are discussed below. 
Vertex (pl. vertices): The fundamental unit of a network, also called a site(physics), anode (computer science), or an actor (sociology).

Edge: The line connecting two vertices. Also called a bond (physics), a link (computer science), or a tie (sociology).

Directed/undirected Edge: An edge is directed if it runs in only one direction (such as a oneway road between two points), and undirected if it runs in both directions. Directed edges, which are sometimes called arcs, can be thought of as sporting arrows indicating their orientation. A graph is directed if all of its edges are directed. An undirected graph can be represented by a directed one having two edges between each pair of connected vertices, one in each direction.

Degree: The number of edges connected to a vertex. Note that the degree is not necessarily equal to the number of vertices adjacent to a vertex, since there may be more than one edge between any two vertices. In a few recent articles, the degree is referred to as the lconnectivity" of a vertex, but we avoid this usage because the word connectivity already has another meaning in graph theory. A directed graph has both an in-degree and an out-degree for each vertex, which are the numbers of in-coming and out-going edges respectively.

Diameter: The diameter of a network is the length (in number of edges) of the longest geodesic path between any two vertices. A few authors have also used this term to mean the average geodesic distance in a graph, although strictly the two quantities are quite distinct.

Degree centrality: A node's (in-) or (out-) degree is the number of links that lead into or out of the node. In an undirected graph they are of course identical. Often used as measure of a node's degree of connectedness and hence also influence and/or popularity

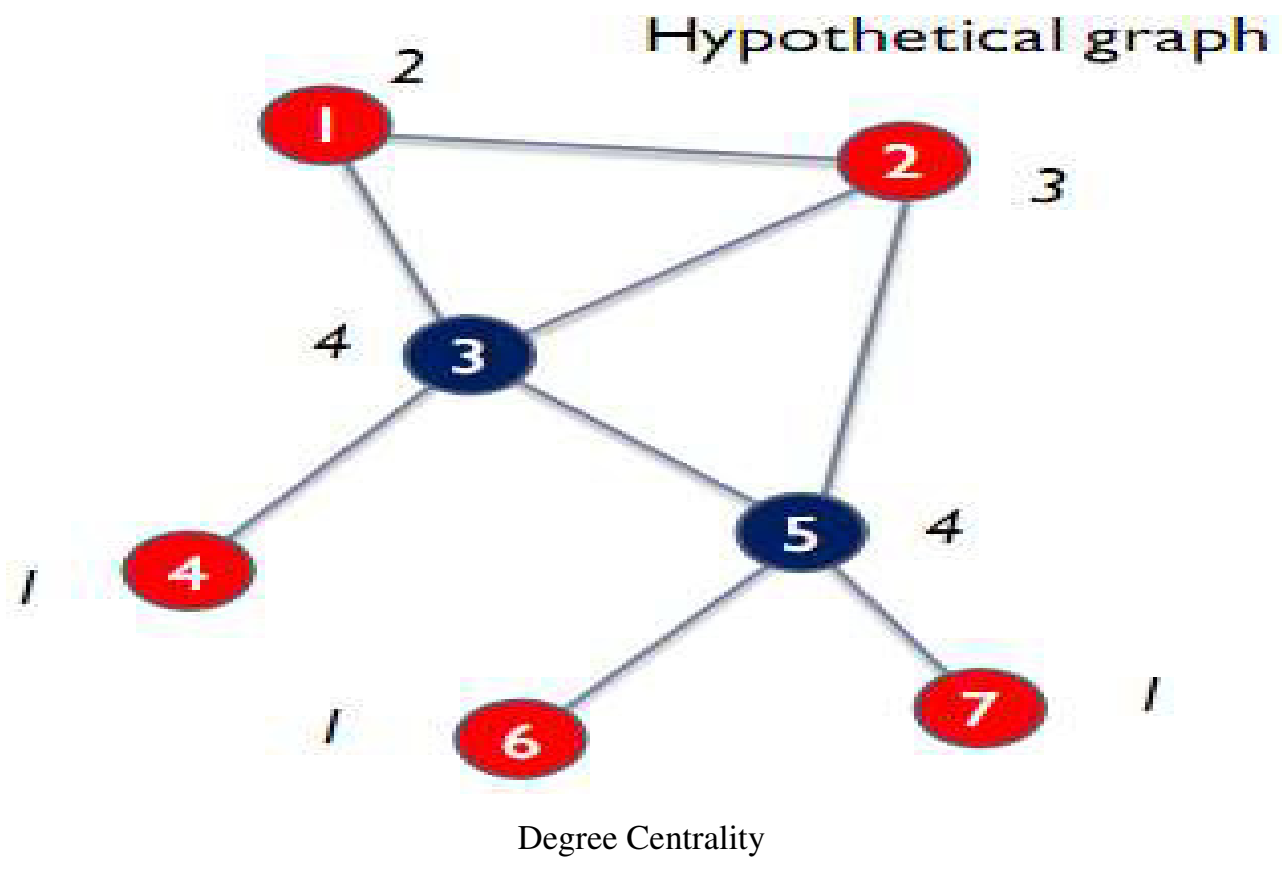


It is useful in assessing which nodes are central with respect to spreading information and influencing others in their immediate _neighborhood".

Betweeness centrality: The number of shortest paths that pass through a node divided by all shortest paths in the network. It shows which nodes are more likely to be in communication paths between other nodes. It is also useful in determining points where the network would break apart.

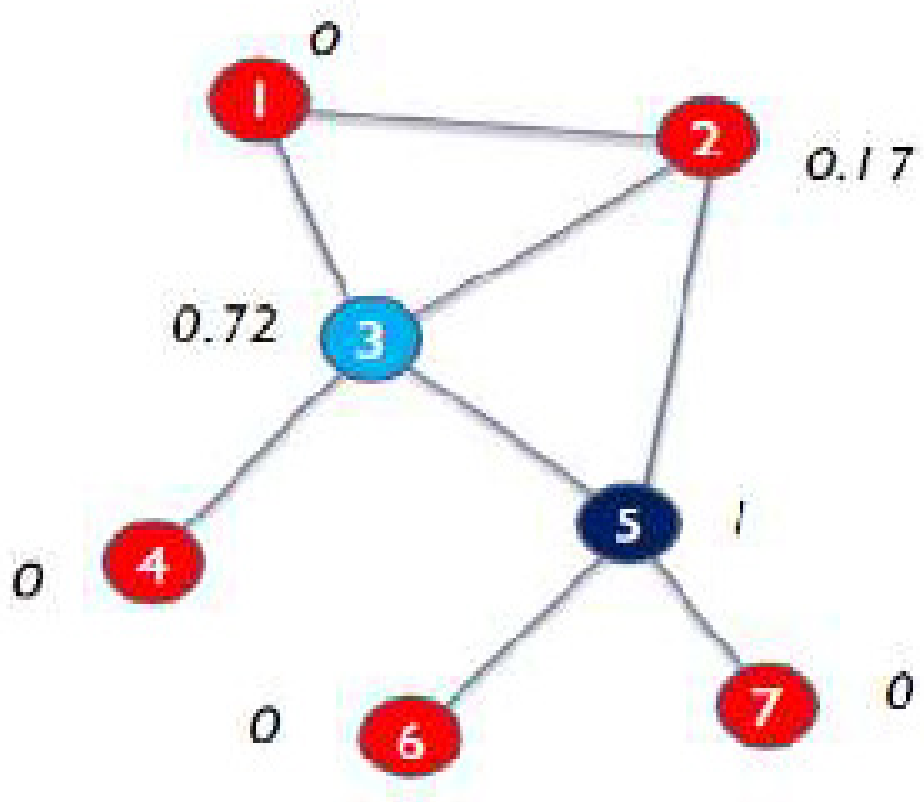

Betweeness centrality

Closeness centrality: The mean length of all shortest paths from a node to all other nodes in the network It is a measure of reach, i.e. how long it will take to reach other nodes from a given starting node.

\section{RECOMMENDER SYSTEM}

Over the last decade, Recommender Systems became an important research area to find out new approaches of recommendation both in industry and academia. The interesting this area still remains high because of the abundance of practical applications that help users to deal with information overload and provide personalized recommendations, content and services to them. Recommender systems can be traced back to the extensive work in the cognitive science, approximation theory, information retrieval, forecasting theories, and also have links to management science, and also to the consumer choice modeling in marketing. Recommender systems or recommendation systems are a subclass of information filtering system that seek to predict the 'rating' or 'preference' that a user would give to an item (such as music, books, or movies) or social element (e.g. people or groups) they had not yet considered, using a model built from the characteristics of an item or the user's social environment. The recommendation problem can be formulated as follows: Let $\mathrm{C}$ be the set of all users and let $\mathrm{S}$ be the set of all possible items 
that can be recommended, such as books, movies, or friends. Let $\mathrm{u}$ be a utility function that measures usefulness of item $\mathrm{s}$ to user c, i.e. $\mathrm{u}: \mathrm{C} \times \mathrm{S} \rightarrow \mathrm{R}$,

Where $\mathrm{R}$ is a totally ordered set (non-negative integers or real numbers within a certainrange). Then for each user $c \in C$, we want to choose such item $s^{\prime} \in S$ that maximizes theuser's utility. More formally:

$\forall \mathrm{c} \in \mathrm{C}, \mathrm{s}^{\prime} \mathrm{c}=\arg \max \mathrm{u}(\mathrm{c}, \mathrm{s})$

In recommender systems the utility of an item is usually represented by a rating,which indicates how a particular user liked a particular item Chaoji, (2006).Generally Recommender systems are divided into two categories.

- Content-based recommendations: the user is recommended items similar to theones the user preferred in the past

- Collaborative recommendations: the user is recommended items that people withsimilar tastes and preferences liked in the past

3.1 Content-based filtering methods are based on information about and characteristics of the items that are going to be recommended. In other words, these algorithms try to recommend items that are similar to those that a user liked in the past(or is examining in the present). In particular, various candidate items are compared with items previously rated by the user and the best-matching items are recommended. The system creates a content-based profile of users based on a weighted vector of item features. The weights denote the importance of each feature to the user and can be computed from individually rated content vectors using a variety of techniques. Simple approaches use the average values of the rated item vector while other sophisticated Methods use machine learning techniques such as Bayesian Classifiers, cluster analysis, decision trees, and artificial neural networks in order to estimate the probability that the user is going to like the item. Content-based approach to recommendation has its roots in information retrieval and information filtering research. Because of the significant and early advancements made by the information retrieval and filtering communities and because of the importance of several textbased applications, many current content-based systems focus on recommending items containing textual information, such as documents, Web sites(URLs), and Usenet news messages. Info finder Abe, (2006) and News weeder Cavalcanti, (2006) are some examples of content-based model. Content-based approach has some limitations like the following:

Limited content analysis: The content must either be in a form that can be parsed automatically by a computer (e.g., text), or the features should be assigned to items manually. Another problem with limited content analysis is that, if two different items are represented by the same set of features, they are indistinguishable. Therefore, since text based documents are usually represented by their most important keywords, content based systems cannot distinguish between a wellwritten article and a badly written one, if they happen to use the same terms.

Over-specialization: The system can only recommend items that score highly against a user's profile; the user is limited to being recommended items similar to those already rated. 
New User Problem: user has to rate a sufficient number of items before a content based recommender system can really understand user's preferences and present the user with reliable recommendations. Therefore, a new user, having very few ratings, would not be able to get accurate recommendations.

3.2 Collaborative Filtering models recommend new items based on previous transactions as well as preference of similar users Anderson, (2007). This method collect and analyze a large amount of information on users ' behaviors, activities or preferences and predicting what users will like based on their similarity to other users. User-based collaborative filtering attempts to model the social process of asking a friend for a recommendation. A key advantage of the collaborative filtering approach is that it does not rely on machine analyzable content and therefore it is capable of accurately recommending complex items such as movies without requiring an "understanding" of the item itself. Collaborative filtering is mainly divided in two categories: memory-based and model-based collaborative algorithms Dumas, (2003). The entire user-product database is used in memory-based algorithms to make a prediction while the modelbased algorithm first generates a model of ratings and then predict. Though this approach has demonstrated its usefulness in many applications, it still has limitations that includes,

New user problem: The system must first learn the user's preferences from the ratings that the user makes.

New item problem: New items are added regularly to recommender systems. Collaborative systems rely solely on users "preferences to make recommendations. Therefore, until the new item is rated by a substantial number of users, the recommender system would not be able to recommend it.

Sparsity: The number of ratings already obtained is usually very small compared to the number of ratings that need to be predicted. Effective prediction of ratings from a small number of examples is important. Also, the success of the collaborative recommender system depends on the availability of a critical mass of us. There are some hybrid models also where content-based and collaborative-based models have been unified to compromise their shortcomings Wolfgang, (2007). They use components like linear combination of predicted ratings, various voting schemes, incorporating one component as a part of the heuristic for the other. For example, $\mathrm{H}$. Kashima \& N. Abe, 2006 uses hybrid recommendation system.

\subsection{Friend Recommendation System}

With the rapid growth of social networks, users of SNSs may easily get overwhelmed by the excessive volume of information. The friendship can significantly affect the quality of recommendations. Therefore, the recommendation of better friend is the essential factor of social network sites to find truly valuable information. There are many friend recommendation systems for social networking sites. Topological characteristics of Social networks have been well researched $[13,14]$ while the theoretical research of complex systems has also grown Dominic, P. D. D., \& Jati, H. (2010,). Silva, N., Tsang, defined the link prediction problem as given a snapshot of a social network at time t, predicting the edges that will be added to the network during the time interval 
Silva, (2010) introduced a new clustering index and user calibration procedure with Genetic Algorithm to suggest friends. Knowledge of the structure and topology of these complex networks combined with quantitative properties such as size, density, average path length or cluster coefficient is used in this approach. Albeit a topology based approach, the innovative approach towards this problem certainly opened a new possibility. Balfanz, $\mathrm{D}(2008)$ proposed the SCAN method to find potential cohesive subgroups which can be used further to friend recommendation. This method was invented in a social hypertext context.

Moreover, Graph based features have also been used in proposing efficient friend recommendation system. L. Katz (2006)proposed WMR- a graph based friend recommendation algorithm. They show weighted minimum-message ratio (WMR) which generates a limited, ordered and personalized friend lists by the real message interaction number among web members. In Collaborative and Structural recommendation of friends Liben-Nowel, (2003) uses the link structure of a social network and content-based recommendation using mutual declared interests. They investigate the problem of link recommendation in such weblog-based social networks and describe an annotated graph-based representation for such networks. It uses graph feature analysis to recommend links $(\mathrm{u}, \mathrm{v})$ given structural features of individual vertices and joint features of the start and end points of a candidate link, such as distance between them. Spiritual and Social context was used by Kwon (2010) to propose a method that measures friendship strength and then suggests friends from a list. The main idea of the proposed method is consisted of the following three stages; (1) computing the friendships core using physical context; (2) computing the friendship score using social context; (3)combining all of the friendship scores and recommending friends by the scoring values.

\section{Proposed System}

In the previous chapter, we have extensively discussed about the existing friend recommendation system. We have tried to find out the problems of that system and gain a lot of information about social networking and friend recommendation. After analyzing those, we also try to make a new system for suggesting friends in social networking sites. In this section, we present our proposed friendship algorithm based on cohesion

\section{Cohesion in Social Community}

Cohesion is an abstract term that is easy to grab by intuition but surprisingly difficult to define in a strict manner. Informally cohesion is the sum of all the factors that attract people to join or to be part of a group. Cohesion refers to the degree to which the elements of a module belong together. Modules with high cohesion tend to be preferable because high cohesion is associated with several desirable traits of software including robustness, reliability, reusability, and understandability whereas low cohesion is associated with undesirable traits such as being difficult to maintain, difficult to test, difficult to reuse, and even difficult to understand. In Social Networking, Cohesion is defined in a connected network and it is considered that network with high degree connectedness is more cohesive. Cohesion is an integral part of physical community and it is assumed that cohesion will have the same impact on social networking. So the impact of the cohesion cannot be ignored in online social networking and it is very effective term to recommending friends for a person in social networking sites. From the definition we can see that cohesion can be thought of two parameters. One is connectedness and another is density. Whenever this parameter comes, the term modularity is suitable to explain cohesion. Because 
modularity is one measure of the structure of networks or graphs. It was designed to measure the strength of division of a network into modules (also called groups, clusters or communities). Networks with high modularity have dense connections between the nodes within modules but sparse connections between nodes in different modules. Modularity is used for detecting community structure in networks with strong cohesion. We have also used this method to finding out cohesive communities in networks for recommending friends.

\section{Frame Work of proposed approach}

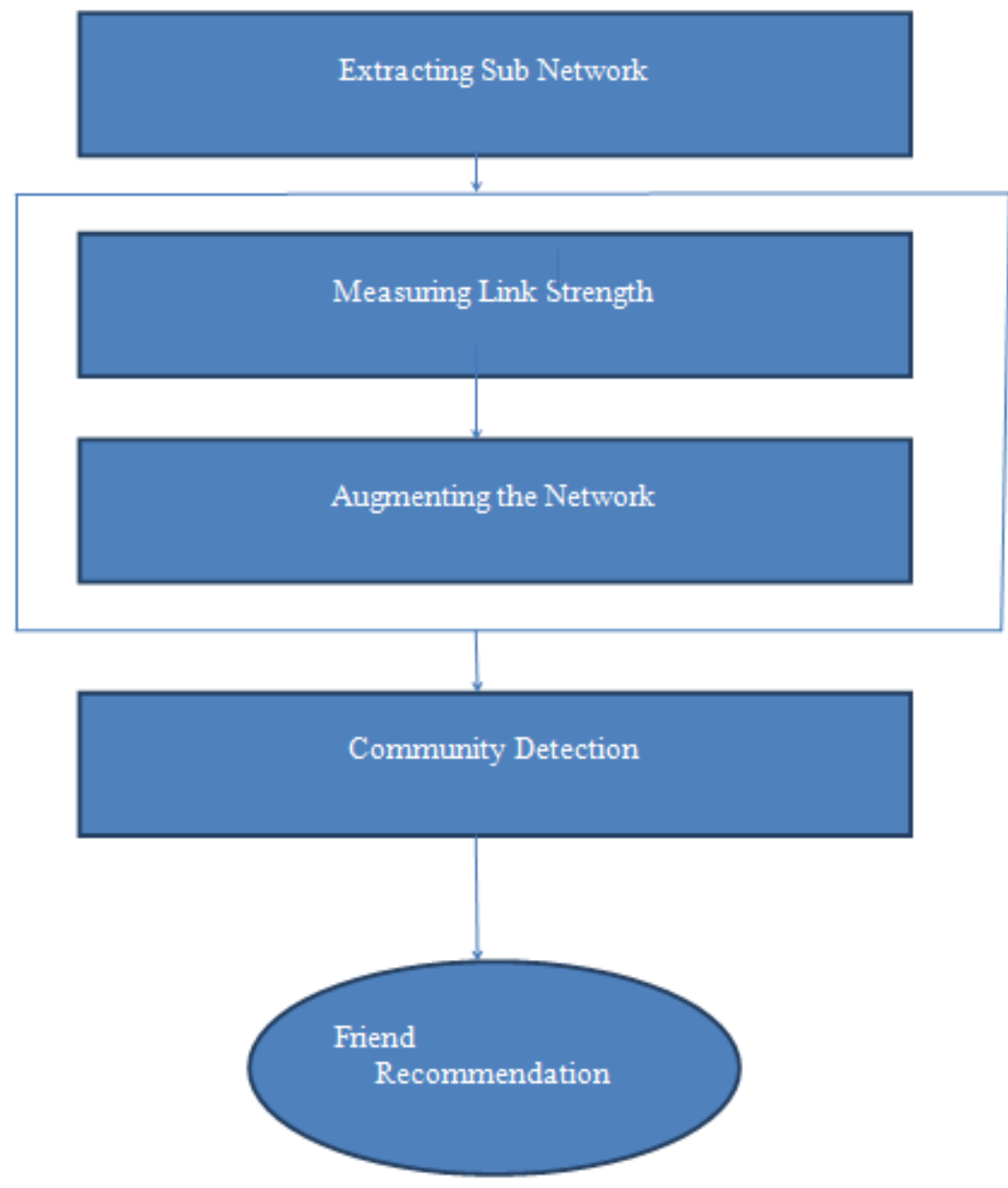

Figure: A Framework of proposed friend recommendation system

\section{DETAIL EXPLANATION OF PROPOSED SYSTEM}

\section{Extracting Sub-network}

Social Networking sites are very large entity with its size. Day by day the size of the network is increasing and as the people are joining there is huge number of information overload happens on those sites 
For experiment of our proposed system, we take the whole network of a random individual. After getting the whole network of a client for who are going to suggest friends, we extract the subnetwork of _x' people from the visualized graph.

\section{Measuring Link Strength}

This is an important step of our proposed friend recommendation system. In this step we first collect data from the users of the extracted graph and requested them to provide our desired data of mutual friend, group information and application use. For measuring the link strength between to users, we will consider these three parameters.

A. Mutual Friend: Mutual friend means common friend. In the social networking sites, as there are several common friends between the users. The traditional social networking sites mainly use this _mutual friend" information to suggest friend. Formally, we can define mutual friend as like, $\mathrm{w}$ is a mutual friend of $\mathrm{u}$ and $\mathrm{v}$, if and only if $\mathrm{w}$ is friends to both $\mathrm{u}$ and $\mathrm{v}$.

mathematically, Mutual $(\mathbf{u}, \mathbf{v}, \mathbf{w})$ I ff f $\mathbf{f}(\mathbf{u}, \mathbf{w}) \wedge \mathbf{f}(\mathbf{v}, \mathbf{w})$

B. Group: In social networking we can see that there are many types of groups for different purposes. People join these groups whenever they feel important on that. Sometimes for professional, ideological and academic reasons people join these group that should count bin calculating the strength between the links/.

C. Application use: People join these type of social network not only for interaction but also they want to get interesting things that can make their time enjoyable. So this social networking sites always try to introduce different application that user can get interest. It is observable that people are using this type of application very frequently.

Formula of measuring link strength with example

We measure the link strength of a client node $\mathrm{T}$ and any node Friend $\mathrm{T} 1$ by the following rules-

$\operatorname{Link}_{\text {strength }}(T, T 1)=$ Weight $_{1} * \mathbf{f 1}(\mathbf{T}, \mathbf{T 1})+$ Weight $_{2} * \mathbf{f}_{2}+$ Weight $_{3} * \mathbf{F}_{3}(\mathbf{T}, \mathbf{T 1}) \mathbf{b}$

Where,

$\mathrm{F} 1(\mathrm{~T}, \mathrm{~T} 1)=$ Number of mutual friends/ Total friends of $\mathrm{T}$

$\mathrm{F} 2(\mathrm{~T}, \mathrm{~T} 1)=$ Number of groups both have joined/ Total groups joined by $\mathrm{T}$

F3 $(\mathrm{T}, \mathrm{T} 1)=$ Number of same apps both use/ Total apps used by $\mathrm{T}$

Weight $_{1}($ mutual firend $)=0.5 ;$ Weight $_{2}($ group $)=0.3 ;$ Weight $_{3}($ App use $)=0.2$

The weights for different parameters have set empirically

Example: subject A has 7 mutual friends with subject B. There are 3 groups where they both have joined and 4 apps they both use. And the total number of friends of $\mathrm{A}$ is 12 . 
The total group joined by A is 5 while A used a Total number of 6 applications.

So, the link strength between A to B can be easily calculated using the rules specified earlier $\operatorname{Link}_{\text {strength }}(\mathrm{A}, \mathrm{B})=0.5 * \frac{7}{15}+0.3 * \frac{3}{5}+0.2 * \frac{4}{6}=0.546$

\section{Augmenting the Network}

After measuring the link strength, we augment the network with links between people that we think can happen but not present in the network. To consider possible links for a client $\mathrm{T}$, we will only take into account his 'friends of friends', thereby creating triads, increasing clustering coefficient. To determine whether a link can happen, we have used the 'Lenient Conjecture' rule.

\section{Lenient Conjecture rule:}

For a client $\mathrm{T}$, a link between him and his friend of friend $\mathrm{T} 2$ can happen if

$\frac{\operatorname{Link}_{\text {strength }}\left(T, T_{2}\right)+\text { Link }_{\text {strength }}\left(T_{2}, T\right)}{2}>$ Threshold

Where,

Threshold $=\frac{\sum_{i=0}^{n} \text { Existing Link }_{\text {strength }(i)} \text { for } T}{n}$

\section{Explanation of 'Linient Conjecture' rule:}

Let, subject A is friend with Subject B \& C. hence, Subject A's Threshold to be friend with another node. So the threshold of A can be calculated by this rule

Threshold $=\frac{\operatorname{Link}_{\text {strength }}(A, B)+\operatorname{Link}_{\text {strength }(A, C)}}{2}=(0.561+0.588) / 2=0.5745$

Now, let, subject $\mathrm{D}$ is a friend of subject $\mathrm{A}$. for $\mathrm{D}$ have to be $\mathrm{A}^{\prime}$ is friend the following condition must be true-

$\frac{\text { Link }_{\text {strength }}(A, D)+\text { Link }_{\text {strength }}(D, A)}{2}>$ Threshold

But here, $(0.478+0.4125) / 2>0.5745 .0 .442$ is not greater than 0.5745 . so the link between $A$ and $\mathrm{D}$ is not possible and we will not augment this connection.

\section{Community Detection \& Friend recommendation:}

In the study of networks, such as social networks a number of different characteristics have been found to occur commonly, including the small-world property, heavy-tailed degree distributions, and clustering, among others. Another common characteristic is community structure. In the context of networks, community structure refers to the occurrence of groups of nodes in a network that are more densely connected internally than with the rest of the network. 
In this step of our proposed system, we detect community in the social networks using the stateof-the-art "Louvaine Method'. This method uses modularity and link strength to detect the community among the networks.

The Louvain method is a simple, efficient and easy-to-implement method for identifying communities in large networks. The method unveils hierarchies of communities and allows to zoom within communities to discover sub-communities, sub-sub-communities, etc. it is today one of the most widely used method for detecting communities in large networks.

The method is a greedy optimization method that attempts to optimize the "modularity" of a partition of the network. The optimization is performed in two steps. First, the method looks for "small" communities by optimizing modularity locally. Second, it aggregates nodes belonging to the same community and builds a new network whose nodes are the communities. These steps are repeated iteratively until a maximum of modularity is attained and a hierarchy of communities is produced.

After detecting community, we will recommend a client people from same communities who are yet to be friends. Also, also for people already friends with all of their neighbors in the same community, we have recommended them with people with the highest Link strength $_{\text {from other }}$ communities.

\section{EXPERIMENT AND RESULT ANALYSIS}

\subsection{Experiment Details}

We experiment our whole proposed system based on the popular social networking site Face book. For extraction of the sub-network, we took the whole Face book network of a random individual and visualize it with industrial-Strength research tool Gephi 0.8.1. Then, we cut off a random sub-network of 10 people.

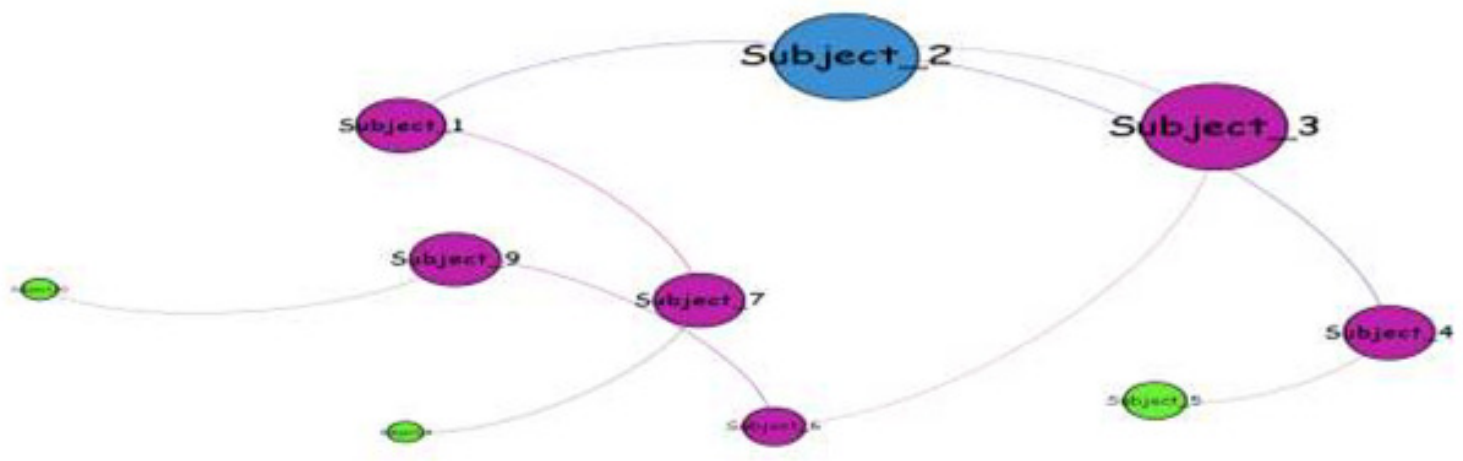

Figure: Extracted sub-network of 10 people

After extraction of sub-network of 10 people we use the roster method to collect our desired data that is needed to calculate link strength. We requested each member of the network to provide the information of mutual friend, groups and application use. 
Some of the data of Subject_2 is shown on the following table.

Table: Data for calculating link strength

\begin{tabular}{|l|l|l|l|l|l|l|}
\hline Connection & $\begin{array}{l}\text { Mutual } \\
\text { friend }\end{array}$ & $\begin{array}{l}\text { Total } \\
\text { friend }\end{array}$ & $\begin{array}{l}\text { Same } \\
\text { group }\end{array}$ & $\begin{array}{l}\text { Total } \\
\text { group }\end{array}$ & $\begin{array}{l}\text { Same App. } \\
\text { Use }\end{array}$ & $\begin{array}{l}\text { Total } \\
\text { App. Use }\end{array}$ \\
\hline $\begin{array}{l}\text { Subject }-2- \\
\text { Subject -3 }\end{array}$ & 8 & 20 & 3 & 8 & 2 & 10 \\
\hline $\begin{array}{l}\text { Subject - 2- } \\
\text { Subject_4 }\end{array}$ & 4 & 20 & 2 & 8 & 4 & 10 \\
\hline $\begin{array}{l}\text { Subject_2 } \\
\text { Subject_1 }\end{array}$ & 9 & 20 & 3 & 8 & 2 & 10 \\
\hline $\begin{array}{l}\text { Subject_2- } \\
\text { Subject_9 }\end{array}$ & 9 & 20 & 5 & 8 & 1 & 10 \\
\hline
\end{tabular}

After collecting data from each users of the network, using perl scripts we calculate the link strength of the connection between existing links. Then we got the result between different links. Some of the results are shown below:

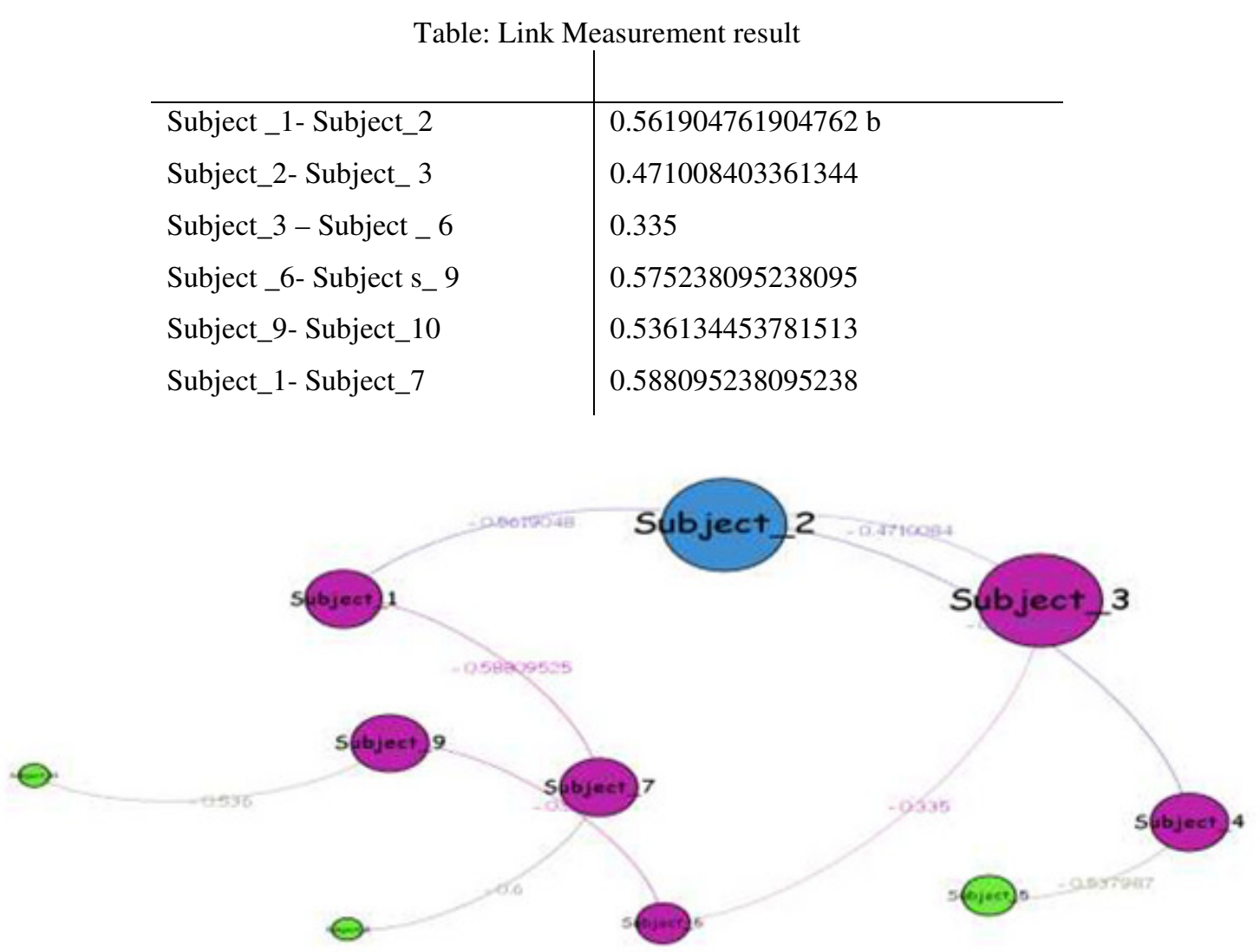

Figure: Link strength connection

After that we augment the network that means we create some connections between different nodes (people) that are not present in the network but yet they can be friend of each other. In case of augmenting the network we do not randomly create a connection. We use the lenient 
conjecture rule that check the possibility of link creation between too connections. For the calculations we took the link strength is greater than the Threshold value then we will create connection and augment the network. If the link strength is less than the threshold value, we do not recommend those links. Some of the results are shown in the following table.

\begin{tabular}{|l|l|l|l|l|l|}
\hline Link & Strength & Strength2 & $\begin{array}{l}\text { Avg. Between } \\
\text { link }\end{array}$ & Threshold & Link Possibility \\
\hline $\begin{array}{l}\text { Subject_1- } \\
\text { Subject_3 }\end{array}$ & 0.561 & 0.588 & 0.578 & 0.4875 & Possible \\
\hline $\begin{array}{l}\text { Subject_1- } \\
\text { Subject_4 }\end{array}$ & 0.1041 & 0.114 & 0.1091 & 0.165 & Not possible \\
\hline $\begin{array}{l}\text { Subject_1- } \\
\text { Subject_8 }\end{array}$ & 0.471 & 0.527 & 0.678 & 0.703 & Not possible \\
\hline Subject_2- & 0.471 & 0.527 & 0.678 & $0 . .334$ & Possible \\
\hline
\end{tabular}

After that getting the augmented network, we applied the state-of-the-art louvaine method that is a simple, efficient and easy-to-implement method for identifying communities in large network. This louvaine method is implemented in different social networking tools. Gephi is one of the tolls we used to implement the louvaine method to detect community.

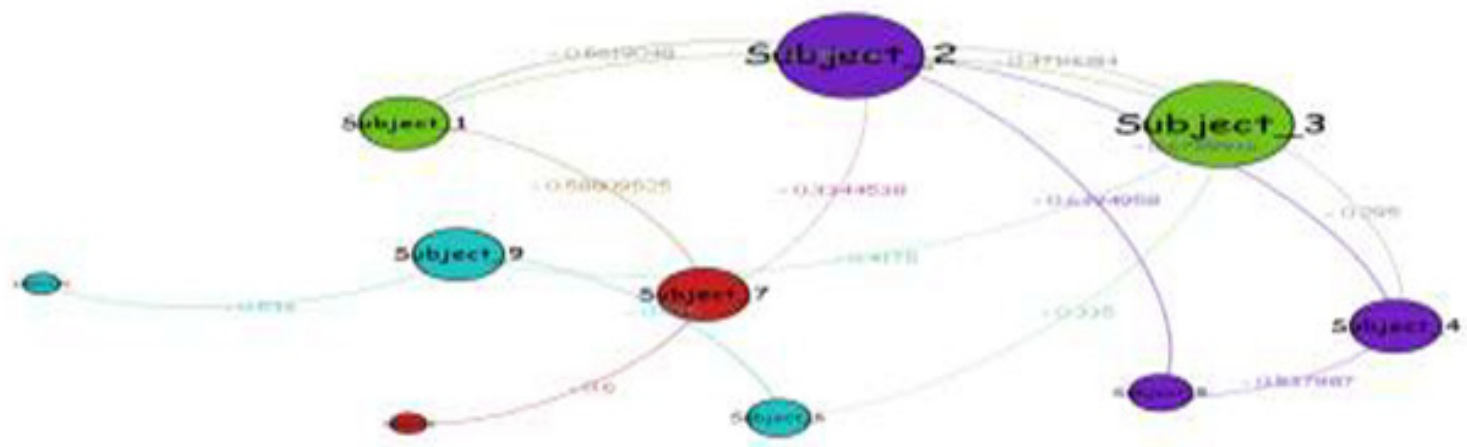

Figure: Community Detection

Using the Gephi tool we detect the community in the networking the figure the same color consist of same community. Then finally we recommend friends within the same community those are yet to be friends. In the time of recommending friends, we check link strength where the link strength is so high they are more cohesive and very good possibility to become friends.

\subsection{Result Analysis}

We have analyzed out result with the use acceptance value and find out the percentage of success. We have also analyses our system using any of the one parameter (mutual friend, group, application use and got the following result. 


\begin{tabular}{|l|l|l|l|l|}
\hline & Mutual friend & Group & App use & All three parameters together \\
\hline Community Detected & 3 & 5 & 3 & 4 \\
\hline Recommended & 8 & 4 & 7 & 14 \\
\hline Accepted & 5 & 3 & 4 & 10 \\
\hline Acceptance Rate & $62.5 \%$ & $75 \%$ & $57.14 \%$ & $71.422 \%$ \\
\hline
\end{tabular}

From the result we can see that if we use only the mutual friend that means the trivial FOF (friend of friend) system then the acceptance rate is less than if we use also group and application use parameter. Though we have experimented our system with a small sub-network because of the unavailability of social networking data, but according to the definition of cohesiveness it is expected that with the combination of this three parameters, the calculation of link strength and recommending friends will be better.

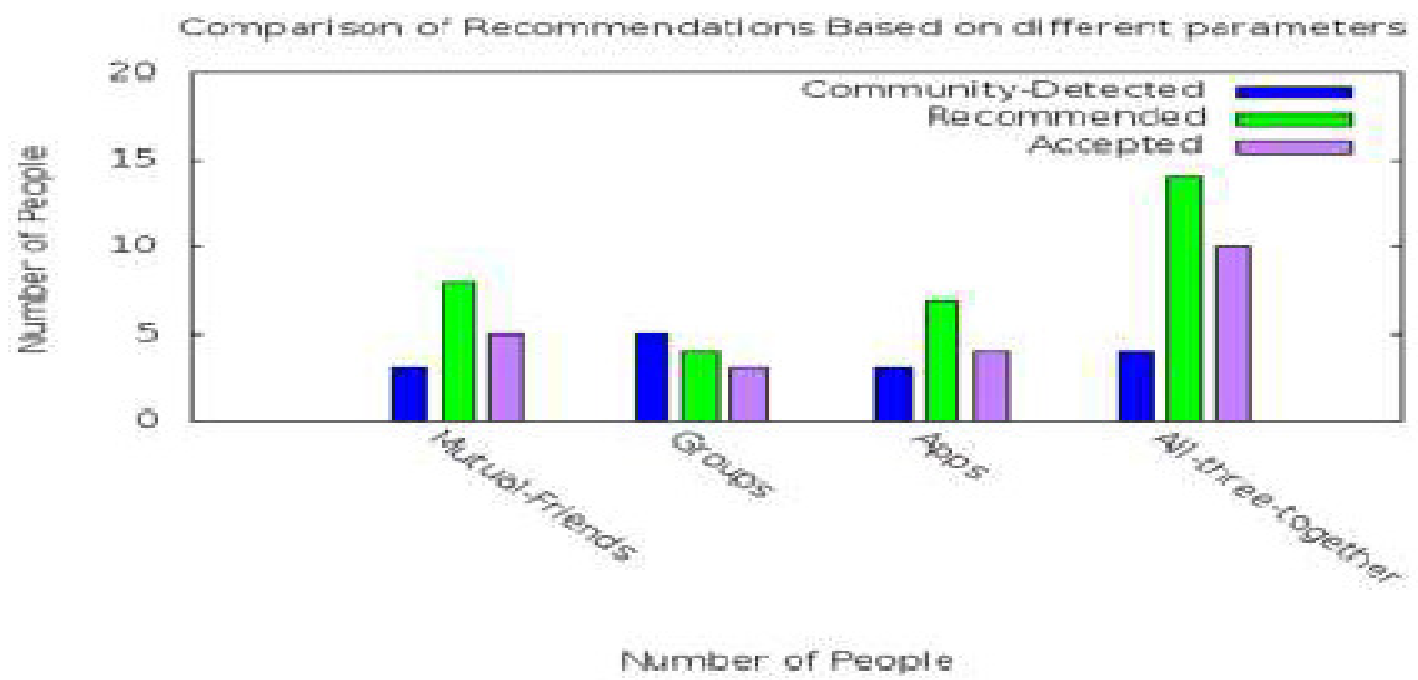

Figure: Result analysis

\section{CONCLUSION AND FUTURE WORK}

With the revolution of web 2.0, social Networking Sites are getting popularity increasingly. People are joining in those sites to share their views to online friends like their real life friends. For this reason, the size and information of these sites are increasingly day by day. Users of these sites hope that the system administrator will provide them recommendation system to make friends. But the recommendation system of human being is not same as the product recommendation as the psychology of human being is different for every person. After realizing this issue, we review the existing system of friend recommendation system in SNSs. And we find that cohesion can be a good measurement in case of social networking recommendation system. So we are proposing this cohesion based friend recommendation system. We hope that this frame work of recommendation system will improve the quality of friend suggestion and will help user to social networking sites. 
Though social networking is now so famous and not that much research has conducted so there are vast scopes to find technique and upgrade the system. For future work, it is important to test the proposed mechanism more intensively in a larger algorithm of friend recommender system. There is also scope on work not to recommend a possible good recommendation a bad one. If the system recommends some people who are not at all related to the user then the user might lose their faith from the sites.

The work we have done can be extended in future. There is a huge scope to improvise this technique a friend recommendation is a new research area and create an application on the proposed system. We have used modularity which has the resolution limit so sometimes it cannot detect small communities in a network. Also using unique parameters, we can improve the robustness of the link strength of the network. We have used a sub-network as our experiment it is extensible to larger network to find out the perfect result of the recommendation system and using a working application.

\section{REFERENCES}

[1] Liben-Nowel, D., And Kleinberg, J, "The Link prediction problem for social networks," Proceedings of the twelfth international conference on Information and knowledge management, pp 556-559. 2003.

[2] Guy, I., Ronen I., and Wilcox E. "Do you know? Recommending people to invite into your social network" Proc. IUI pp. 77-86. 2009.

[3] Kwon, J. and Kim, S. "Friend Recommendation Method using Physical and social Context". IJCSNS International of Computer Science and network security, VOL. 10 No.11, November 2010.

[4] Wolfgang Woerndl and Georg Grog, "Utilizing Physical and Social Context to Improve Recommender systems". In WI- IATW '07: Proceedings of the 2007 IEEE/WIC/ACM International Conferences on Web Intelligence and Intelligent Agent Technology - Workshops, pp. 123-128, 2007.

[5] M. Hasan, V. Chaoji, S. Slem, and M. Zaki. "Link Prediction using supervised learning." In Workshop on Link Analysis, Counterterrorism and Security (SDM), 2006.

[6] H. Kashima and N. Abe. "A parameterized probabilistic model of network evolution for supervised link prediction.” In ICDM ’06, 2006.

[7] L. Katz. "A new ststus index derived from sociometric analysis." Psychometrika, 18:39-43,1953.

[8] Silva, N.,Tsang, I., Cavalcanti, G., and Tsang, I.” A Graph-Based Friend recommendation System Using Genetic Algorith". WCCI 2010 IEEE World Congress on Computational Intellegence July, 1823, 2010-CCIB Barcelona, Spain CEC.

[9] Chiew, T. K., \& Salim, S. S. (2003). Webuse: Website usability evaluation tool. Malaysian Journal of Computer Science, 16(1), 47-57.

[10] Chew, M., Balfanz, D. \& Laurie, B. 2008. (Under)mining Privacy in Social Networks

[11] Donghee Yvette Wohn, The "S" in Social Network Games: Initiating, Maintaining, and Enhancing Relationships, Michigan State University, USA, 2011. 
[12] Dominic, P. D. D., \& Jati, H. (2010, June). Evaluation method of Malaysian university website: Quality website using hybrid method. In Information Technology (ITSim), 2010 International Symposium in (Vol. 1, pp. 1-6). IEEE.

[13] Dumas, J. S. 2003. User-based evaluations. The human-computer interaction handbook: fundamentals, evolving technologies and emerging applications. L. Erlbaum Associates Inc

[14] Dix, A., E. Finlay, J., D. Abowd, G. \& Beale, R. 2004. Human-Computer Interaction, 3rd Edition

[15] Anderson, T. \& Garrison, D. R. (2007). Learning in a networked world: New roles and responsibilities. In Gibson, C. (Ed.), Distance Learners in Higher Education. 